\title{
BMJ Open Patient journey following lumbar spinal fusion surgery (LSFS): protocol for a multicentre qualitative analysis of the patient rehabilitation experience (FuJourn)
}

Alison Rushton, ${ }^{1} \mathrm{~J}$ Bart Staal, ${ }^{2}$ Martin Verra, ${ }^{3}$ Andrew Emms, ${ }^{4}$ Michael Reddington, ${ }^{5}$ Andrew Soundy, ${ }^{1}$ Ashley Cole, ${ }^{6}$ Paul Willems, ${ }^{7}$ Lorin Benneker, ${ }^{8}$ Annabel Masson, ${ }^{1}$ Nicola R Heneghan ${ }^{1}$

To cite: Rushton A, Staal JB, Verra M, et al. Patient journey following lumbar spinal fusion surgery (LSFS): protocol for a multicentre qualitative analysis of the patient rehabilitation experience (FuJourn). BMJ Open 2018;8:e20710. doi:10.1136/ bmjopen-2017-020710

- Prepublication history and additional material for this paper are available online. To view these files please visit the journal online (http://dx.doi. org/10.1136/bmjopen-2017020710).

Received 20 November 2017 Accepted 23 November 2017

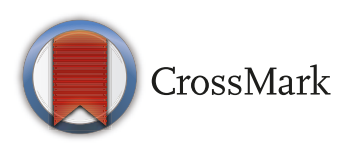

For numbered affiliations see end of article.

Correspondence to

Dr Alison Rushton;

a.b.rushton@bham.ac.uk

\section{ABSTRACT}

Introduction There has been a $65 \%$ increase in lumbar spinal fusion surgery (LSFS) worldwide over the last 13 years, with costs of $£ 26$ million to the UK National Health Service annually. Patient dissatisfaction with outcome and persistent pain and disability incurs further costs. Three trials provide low-quality evidence for the role of physiotherapy. Our UK surveys investigating physiotherapy/surgeon practice concluded rehabilitation should be tailored to the individual patient owing to considerable clinical heterogeneity. This study will explore the perceptions of patients who undergo LSFS to inform precision rehabilitation.

Methods and analysis A qualitative study, using interpretive phenomenological analysis, will recruit a purposive sample $(n=40)$ to ensure patterns of similarity and difference in their journeys can be explored. Indepth semistructured interviews will be undertaken following discharge from hospital and at 12 months postsurgery. Patients' preoperative and postoperative experiences, underlying attitudes and beliefs towards the surgical intervention, facilitators and barriers to recovery, adherence to advice and physiotherapy, experiences of rehabilitation and return to normal function/activity/work will be explored. A 12-month patient diary will provide real time access to patient data, capturing a weekly record of life as lived, including symptoms, medication, experiences of stages of recovery, rehabilitation adherence, healthcare professional appointments, attitudes, their feelings and experiences throughout their journey. Data will be analysed in a number of stages in accordance with interpretive phenomenological analysis, supported using NVivo software. Analysis of the first interviews and patient diaries will afford a rich density of data to build an overall understanding of the patients' lived experiences, informing the 12-month interview. Strategies (eg, reflexivity) will ensure trustworthiness.

Ethics and dissemination The study has ethical approval (IRAS 223283). Findings will ensure that patient-driven data inform precision rehabilitation by understanding the patient journey. Findings will be disseminated through peer-reviewed journals and conferences.
Strengths and limitations of this study

- Qualitative methods are essential to generate indepth understanding of the patient journey, thereby ensuring the research is informed by the people themselves, working with patients, not 'on' them

- The study design was informed by the Consolidated Criteria for Reporting Qualitative Research [COREQ] to ensure a rigorous methodology.

- A purposive sample of $n=40$ will ensure issues of considerable clinical heterogeneity are represented across participants.

- Analysis of the first interview and patient diary will afford a rich density of data, to build an overall understanding of the patients' lived experiences; informing the 12-month interview.

- Patient and public involvement [PPI] is an active component of the study management team to ensure the patient perspective is central to the study.

\section{INTRODUCTION}

Surgery

In the UK, surgery is the greatest single component of expenditure for managing low back pain, ${ }^{1}$ with 4500 lumbar spinal fusion surgery (LSFS) procedures performed annually. ${ }^{2}$ However, a further 73000 procedures were recorded as 'other operations on the spine', and accurate data are therefore lacking. LSFS is increasingly used for degenerative lumbar spine problems, although low-level current evidence suggests inconclusive outcomes and questions cost-effectiveness. ${ }^{3}$ From current data, ${ }^{2}$ a $65 \%$ increase in LSFS (also illustrated internationally, although data reveals considerable variation in rates between regions within and between countries ${ }^{4}$ ) over 13 years costs the National Health Service (NHS) $£ 26$ million annually. LSFS accounted for 
$14 \%$ USA spending on back surgery in 1992, increasing to $47 \%$ by $2003 .{ }^{4}$ Contradicting this increase, the recent UK National Institute for Health and Care Excellence guideline $^{5}$ is unequivocal in recommending first that LSFS should only be offered for patients with non-specific low back pain [NSLBP] if being investigated in a randomised controlled trial, and second that further research should investigate whether LSFS should be a management option for patients with NSLBP.

A recent survey from the Netherlands identified a lack of consensus between surgeons $(n=62)$ regarding prognostic factors and predictive tests aiding patient selection for LSFS, ${ }^{6}$ contributing to a broad clinical heterogeneity of patients undergoing LSFS. Additionally, our survey of UK current practice found variation between surgeons regarding surgical procedure, for example, open or minimally invasive, instrumented or non-instrumented, different approaches, for example, transforaminal, and different numbers of levels fused, ${ }^{7}$ dependent on the individual patient and their indications for surgery.

There is minimal knowledge regarding long-term outcome $^{1}$ following LSFS. Swedish National Spine Register data identified that $25 \%$ of patients had no change or worsened back and/or leg pain following LSFS with $40 \%$ reporting dissatisfaction with their 12-month outcome. ${ }^{8}$ A recent paper ${ }^{9}$ found that across three trials at long-term follow-up (mean of 11 years, range $8-15$ years), there was no difference in outcome between LSFS and a multidisciplinary cognitive behavioural therapy and exercise intervention (mean adjusted difference in treatment effect of -0.7 points on 0-100 Oswestry Disability Index). However, only disability outcomes were investigated, ${ }^{9}$ and long-term outcome remains unclear.

Rehabilitation following LSFS is also therefore of interest. Two recent systematic reviews ${ }^{10}{ }^{11}$ identified three randomised controlled trials affording inconclusive, very low-quality evidence for the effectiveness of physiotherapy rehabilitation. Our evaluations of current UK practice identified extensive variability in who receives physiotherapy and the content of rehabilitation interventions due to the clinical heterogeneity of the LSFS population both presurgery and postsurgery. $^{712}$ Rehabilitation should therefore be tailored to the individual patient (precision rehabilitation). ${ }^{7}{ }^{12}$ The patient's experiences and perceptions are central to this issue and, to date, no research has focused on the patient's journey through LSFS and rehabilitation/recovery. Understanding the patient journey is important to determine best practice.

\section{Rationale}

This study will explore the perceptions of patients who undergo LSFS through a qualitative study focused to the patient's journey. Through semistructured interviews and patient diaries, the patient's individual experience of LSFS and their recovery following surgery will be explored. Qualitative methods are essential to generate in-depth understanding of the patient journey to ensure the research is informed by the people themselves, to work with patients and not 'on' them.

\section{METHODS AND ANALYSIS}

Theoretical framework

Interpretive Phenomenological Analysis (IPA) approach.

\section{Aim}

To explore the perceptions of patients who undergo LSFS in relation to their experiences presurgery and postsurgery, capturing their lived experiences.

\section{Objectives}

1. To explore the experiences of patients and perceptions of their back (and leg) problem, capturing the strategies/mechanisms patients use to cope and manage, from their onset of problems, through decision for surgery and recovery.

2. To understand the patient journey through to their return to function/activity/work as appropriate to the individual.

3. To identify and describe the stages and components of the patient's journey from their own perspective.

4. To explore patterns/relationships within/between contextual groups of patients.

5. To understand the value of physiotherapy rehabilitation for patients, its role in preventing further episodes of spinal pain and adherence to management.

6. To ensure that patient-driven data informs precision rehabilitation and its evaluation by understanding the patient journey.

Additionally, the patients involved in this study will be asked to document their own patient and public involvement (PPI) experience in order to share good practice and improve future work.

\section{Design}

Qualitative study using an IPA approach ${ }^{13} 14$ with multiple sources of data, ${ }^{15}$ informed by the Consolidated Criteria for Reporting Qualitative Studies. ${ }^{16}$ The importance of measuring success of treatment by results that really matter to patients has been driven by the UK Department of Health. ${ }^{17}$ It is now widely acknowledged that qualitative research is of considerable value to improve understanding of the patient experience and the complexity of processes involved in treatment outcome. ${ }^{18}$ Increasingly, qualitative studies are published and inform NHS planning and policy making. As the aim of IPA is to understand experiences from the individual's perspective, ${ }^{13}{ }^{14}$ it is highly appropriate for this study. IPA enables an exploration of what participants make of their experiences and seeks to understand the meanings that the experiences hold, as well as enabling patterns and relationships to be explored. ${ }^{14}$ This is particularly important owing to the considerable clinical heterogeneity of the LSFS 


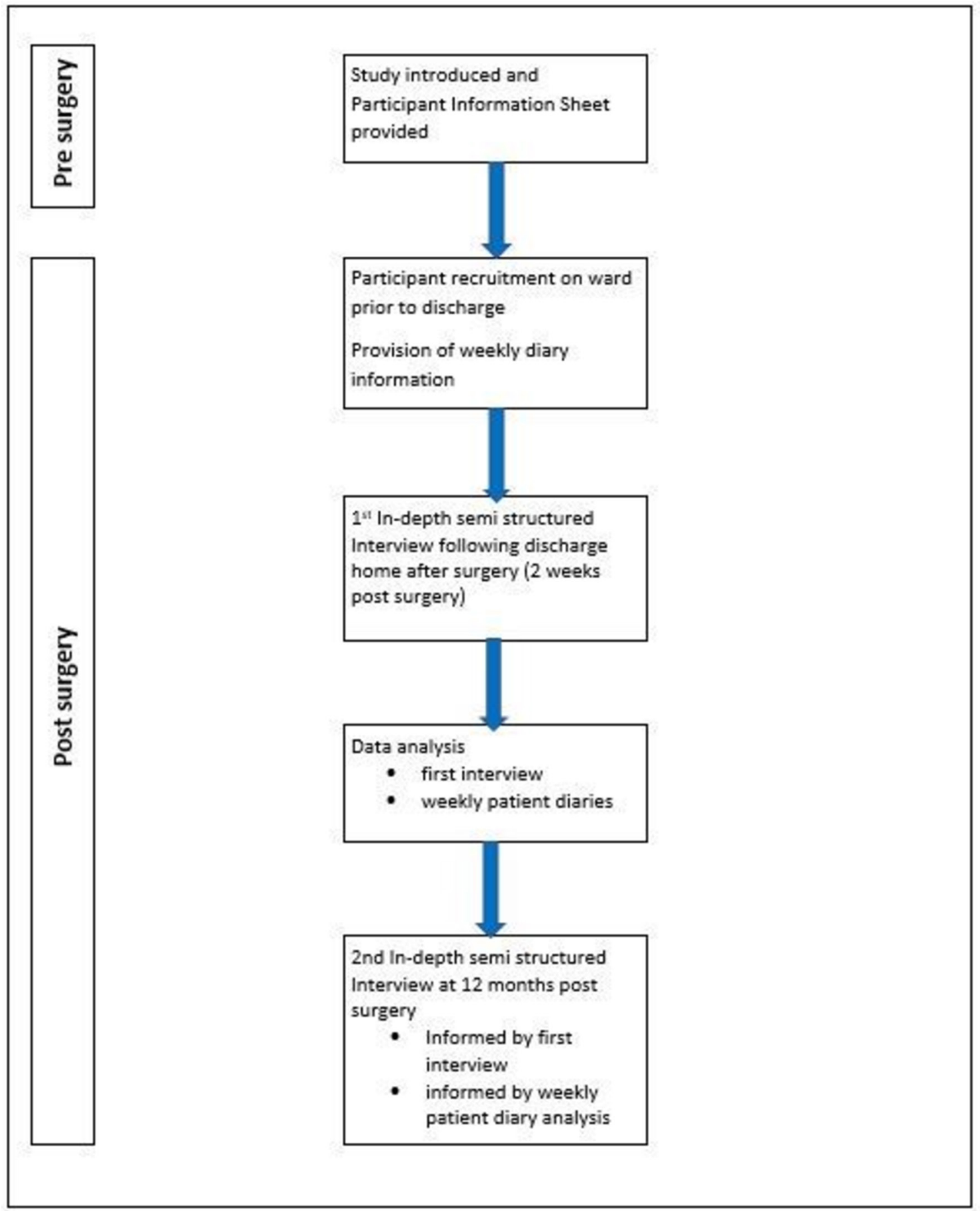

Figure 1 Study flow chart.

population that creates variability of surgical approach and a rehabilitation challenge.

\section{Study setting}

Two UK clinical sites (Royal Orthopaedic Hospital, Birmingham, and Sheffield Teaching Hospitals NHS Trust) in a secondary care setting.

\section{Methods}

In-depth semistructured interviews

Participants will be invited to participate in two in-depth semistructured interviews. The first will be following surgery when the patient has returned home and the second at 12 months postsurgery to ensure early and late components of the patient's journey are captured (see figure 1). The second interview will coincide with the routine 12-month follow-up visit wherever possible to avoid a further journey/organisation of a visit for participants. Interviews with consenting participants will be held at a convenient time and place for the participant; often patients prefer to be interviewed at home, but a NHS Trust site-based location will also be offered. Travel and parking will be reimbursed for 
any patient attending interviews at the Trust. Interviews will be undertaken by an experienced qualitative researcher (AM) who will adhere to the NHS lone working guidance for best practice. Following informed consent, a topic guide will inform the first interviews to explore the patient's individual journey. The topic guide was developed by the research team including our two patient coinvestigators (online supplementary file 1). The guide was constructed using our systematic review and surveys of current practice, ${ }^{7} 1012$ the structure of the patient diary and from the insights of our two patient coinvestigators. The topic guide includes questions regarding a participant's preoperative and postoperative experiences, underlying attitudes and beliefs towards the surgical intervention, expectations from surgery, facilitators and barriers to recovery, adherence to advice and physiotherapy, experiences of rehabilitation and return to normal function/activity/ work, aiming to prompt and capture their individual journey. Participants will be invited to introduce new topics and/or issues into the interviews. We predict from prior experience that interviews will last approximately $60 \mathrm{~min}$. The development of the topic guide is iterative, with the guide for second interview having evolved from analysis of the first interview and patient diary. Prior to the interview, the participant will have spoken to the interviewer and will be aware of the focus of the research, and so existing knowledge and relationships will have been established. The interviewer will spend time before and after the interviews engaging the participant in everyday conversation to put them at ease, also allowing the interviewer to check their well-being. In the unlikely event of distress occurring, appropriate action will be taken by stopping the interview and establishing further participant support if indicated. Participation is entirely voluntary, and the interview can be stopped at the request of the interviewee at any point.

Field notes will be recorded throughout interviews, and a reflexive diary will be used by the interviewer. Interviews will be audio recorded using an encrypted data recorder and transcribed verbatim. Through the process of 'member checking', participants will be afforded the opportunity to read their transcript and add any further reflections, a process that will test the interviewer's interpretation. ${ }^{19}$ The process will be completed through post or email depending on participant preference, with participants also offered the opportunity to discuss by telephone/Skype.

\section{Twelve-month written or electronicpatient diary}

The diary will capture the patient's journey by providing real-time access to participant data, tracking the course of their experiences over time. It will be introduced at recruitment and explained and will capture a weekly record of life as lived, including symptoms, medication, experiences of stages of recovery, rehabilitation adherence, healthcare professional appointments, attitudes and their feelings throughout their journey (online supplementary file S2).

The literature is conflicting on the use of patient diaries to collect longitudinal patient data, not with regards to their value, but to obtain adherence and quality data. In a recent systematic review, four of the seven included studies assessed patient preference, which was for electronic rather than paper data collection. ${ }^{20}$ This is consistent with our recent focus groups ${ }^{21}$ that found polar views in a population postspinal surgery for electronic versus paper data interaction/datacollection. Evolution in personal mobile and tablet technology now enables easy and high-quality audio ${ }^{22}$ collection of data. Consequently, participants will choose either a structured paper/email or audio (using their existing mobile/tablet technology) diary, ${ }^{22}$ requiring a weekly entry. Strategies to enhance diary adherence will include a weekly electronic (text or email) or telephone prompt according to preference to remind completion, and monthly diary collection (post or email) to evaluate ongoing adherence and enable discussion of progress. As a small incentive, a financial incentive for completion of all entries will be provided ( $£ 25$ for completing all diary entries).

\section{Study participants}

To enable a rich in-depth account, an IPA approach advocates the recruitment of participants from a fairly homogenous population for whom the research question holds significance. ${ }^{13}$ This is balanced by the sample size needing to be sufficiently large to enable collection of data relevant to the question to develop a full and interesting interpretation of the data, enabling the exploration of patterns and relationships within and between conceptual groups of participants. ${ }^{23}$ A purposive sample (up to $n=40$ ) of patients undergoing LSFS will be recruited to ensure patterns of similarity and difference in their journeys can be explored, capturing conceptual diversity and not quantification. ${ }^{23}$ The sample size will ensure a range of indications for surgery, ages, gender and ethnicity, ${ }^{6}$ and known predictive factors of outcome, for example, psychosocial factors, educational background, number of levels fused ${ }^{3}$ are captured; thus, reflecting the considerable clinical heterogeneity of this population and their outcomes. We know this clinical heterogeneity, but need to know the similarities and differences in patient's individual journeys to understand the implications of it for recovery. IPA research has previously used this number of participants (in studies of 1-48 participants ${ }^{23}$ ]. The purposive sampling approach will be constantly reviewed and altered if findings indicate. This approach will ensure a density of evidence is created.

Participants at two UK clinical sites (Royal Orthopaedic Hospital, Birmingham, and Sheffield Teaching Hospitals NHS Trust) will be asked by the site physiotherapy lead to consent to face-to-face interviews at two time points: following discharge home after their surgery and at 12 months postsurgery. Patients undergoing their NHS surgery at a private hospital as part of waiting list 
initiatives will be eligible for recruitment. Recruiting up to $\mathrm{n}=20$ at each site is well within annual feasibility data (>100 operations). The diary will be introduced and explained at recruitment to enable participants to familiarise themselves with it in advance of the first interview. It is anticipated that 40 participants will provide rich insight into the patients' journeys, although the precise number of participants, and their characteristics will be determined during the study. Recruitment will continue until the researchers/steering group agree that rich insight is obtained and that further data would not add to our understanding.

\section{Eligibility criteria for the cohort study (and therefore these participants)}

Inclusion criteria: adult patients ( $\geq 16$ years) undergoing up to four-level instrumented LSFS for back pain and/or leg pain of degenerative cause, inclusive of isthmic spondylolisthesis, willing to consent and able to communicate in English.

Exclusion criteria: LSFS for traumatic or pathological spinal fracture, malignancy, infection, deformity requiring more than four-level instrumentation and revision fusion surgery and poor English/communication difficulties.

\section{Sample identification}

Potential participants will be identified by the site lead in discussion with the consultant orthopaedic surgeons and their respective team (registrar, fellow, physiotherapist and nurse) in their outpatient orthopaedic clinic, as all surgery will be elective (ie, not emergency). Posters in clinics will serve as a reminder to the team of the study and will also encourage patients to raise questions about the study. The site lead will also monitor waiting lists and discuss with the booking clerk for potential participants. The patient will attend the orthopaedic clinic to be consented up to 6 weeks prior to their surgery where a tentative date for surgery will be given. At this consenting for surgery appointment, the participant information sheet will be given to the patient, and any questions will be answered by the site lead or recruiting research nurse. The patient will be asked for permission to contact them approximately 2 weeks prior to surgery to discuss any questions they may have regarding the study. The site lead or recruiting research nurse will contact the patient by telephone and further discussion regarding the study will occur at this point and any further questions answered.

\section{Consent}

Consent to participate in the study will be taken either preoperatively or postoperatively prior to discharge depending on patient preference. In this way, the patient is not inconvenienced by return trips to hospital and costs/time incurred as a result. Consent for the study will be taken by the site lead or recruiting research nurse, who are both experienced in research and consent and have current good clinical practice (GCP) training. The site lead or recruiting research nurse will have the necessary experience and skills to assess capacity of the patient to provide informed consent.

\section{Data analysis}

IPA embraces data analysis by considering the meaning of experience. The interview data will be analysed primarily by the physiotherapist conducting the interviews (AM). During the initial stages of data-driven analysis, AM will attempt to suspend all judgements and presuppositions. ${ }^{24}$ However, we note that IPA is a flexible inductive approach, ${ }^{23}$ well suited to studies that relate findings to biopsychosocial theories that often dominate healthcare settings. ${ }^{25}$ For example, investigator AS often uses theories considering motivation, for example, self-determination theory, and concepts from practice, for example, shared decision making, within his analysis as a priori concepts, and thus we may use this within the latter stages. We will adopt a four-stage approach. ${ }^{24}$

\section{Stage 1}

Researcher AM's (and blind reviewer AR for a sample) first encounter with the transcribed text and audio recordings. Transcription will be verbatim including detail of verbal and non-verbal content, for example, speech dynamics. ${ }^{24}$

\section{Stage 2}

Preliminary themes will be identified and presented first to investigator AS, and then the steering group (including patient coinvestigators) for discussion. Data will be coded in accordance with IPA. ${ }^{13}$ An initial phase of the first six interviews will enable the data analyses, purposive sampling and topic guide to be discussed by the steering group and altered if required.

\section{Stages 3 and 4}

$\mathrm{AM}$ and blind reviewer will group themes together as clusters and tabulate in a summary table, illustrated by verbatim extracts. ${ }^{23}$ Data management will be supported through NVivo software. Coinvestigator AS will critique with discussion to consider for a priori concepts. Themes in summary table will be discussed with patient coinvestigators and then wider steering group.

Strategies to ensure trustworthiness will include considering data to the detail of minor themes, blind coding from three experienced researchers (AM, AS and AR), peer and patient critique and review, code-recode audits, a constant comparative process, acknowledgement of the researchers' preconceptions and beliefs and active reflexivity to enable greater transparency. ${ }^{1523}$ A collaborative approach to the analysis representing professional and PPI perspectives aims to enhance researcher reflexivity and hence quality of the analysis. ${ }^{15}$

A further valuable source of data, ${ }^{24}$ the patient diaries, will be analysed by AM and AR with oversight by coinvestigator AS and discussion with the steering group. AM will transcribe the audio entries verbatim. The same stages of analysis in accordance with IPA will be used to analyse the 
diary data. These data will be incorporated into the analysis as identified above, to afford a rich density of data, and will inform the second interviews.

\section{Implications of results}

The outcome of this study will be to ensure that patientdriven data inform decisions regarding surgery and precision rehabilitation by understanding the patient journey, capturing the strategies/mechanisms patients use to cope/manage from their onset of problems through decision for surgery and recovery.

\section{Research governance}

The study will be conducted in accordance with the principles of the Research Governance Framework for Health and Social Care. The study will follow GCP and predefined protocols. Anonymised data will be stored securely. The chief investigator (AR) chairs a study management group comprising the coinvestigators who will meet regularly face to face online to monitor progress and address any issues. The study steering group (SSG) is established already and brings together European experts, representing physiotherapists, surgeons and patients across three countries who are ideally positioned to guide the study and also to explore cross cultural issues identified from the same study running in three European countries. The SSG will overview the study and analyses to inform data interpretation. They will also decide how the patients' journeys will inform further research to inform precision rehabilitation. Anonymised data will be stored confidentially for 10 years in accordance with Data Protection Act and University of Birmingham's research governance frameworks. A low risk to this study is not recruiting enough participants at each site. If this occurs, we will use a further local site.

\section{Patient and public involvement}

This study is coproduced by patients and healthcare professionals. It was conceived directly as a result of patients' comments to the study team regarding their experience of LSFS. Patients post-LSFS are part of our research team/coinvestigators to ensure the patient perspective is central. They have been involved since inception of this programme of research starting $>3$ years ago and have contributed to our understanding of the findings from the systematic reviews informing this application. Patients have contributed to the development of the interview topic guide, diary design, participant information sheet and consent form and will contribute importantly to the processes of data analysis and interpretation and producing a lay summary of findings. They have reviewed this protocol and have helped to ensure that their involvement is fully considered. The patients involved in this study will be asked to document their own 'PPI' experience in order to share good practice and improve future work. Coinvestigator $\mathrm{NRH}$ is the dedicated lead for user involvement and is already working collaboratively with them to ensure appropriate training, support and involvement; the Guidance for Reporting Involvement of Patients and the Public version 2 short form (GRIPP-2SF) is used in reporting PPI. ${ }^{26}$

\section{Ethics and dissemination}

There are minimal risks associated with this study. A potential risk is that while undertaking an interview, the researcher may obtain information concerning the wellbeing of the participant. Mechanisms for safeguarding the participant would be implemented in this situation and include discussion (with permission from the participant) with the site lead physiotherapist to mitigate harm to the participant. Protocol deviations, non-compliances or breaches are departures from the approved protocol. Accidental protocol deviations can happen at any time. They will be adequately documented on the relevant forms and reported to the chief investigator (AR) and sponsor (University of Birmingham) immediately. Deviations from the protocol that are found to frequently recur are not acceptable and will necessitate immediate action and could potentially be classified as a serious breach. All investigators and study site staff will comply with the requirements of the Data Protection Act 1998 with regards to the collection, storage, processing and disclosure of personal information and will uphold the Act's core principles. Personal information will be collected and stored electronically on a password-protected computer at the University of Birmingham. Data will be coded, and depersonalised data with the participant's identifying information will be replaced by an unrelated sequence of characters. Secure maintenance of the data will ensure that the linking code in kept securely in a separate location using encrypted digital files within password-protected folders and storage media. Only the chief investigator and the researcher carrying out the interviews will have access to the data as necessary for quality control, audit and analysis. The confidentiality of data will be preserved when the data are transmitted to sponsors and coinvestigators by maintaining the depersonalised data format and ensuring that no data are traceable to an individual participant. The data custodian will be the chief investigator.

\section{Peer review}

The study protocol has been independently peer reviewed to support funding by the Chartered Society of Physiotherapy-specifically the Physiotherapy Research Foundation's Scientific Committee. This has afforded independent, high-quality and proportionate peer review.

\section{Author affiliations}

${ }^{1}$ Centre of Precision Rehabilitation for Spinal Pain (CPR Spine), University of Birmingham School of Sport Exercise and Rehabilitation Sciences, Birmingham, UK ${ }^{2}$ Scientific Institute for Quality of Healthcare, RadboudUMC, Nijmegen, Gelderland, The Netherlands 
${ }^{3}$ Department of Physiotherapy, Bern University Hospital, Bern, Switzerland ${ }^{4}$ Department of Physiotherapy, The Royal Orthopaedic Hospital NHS Foundation Trust, Birmingham, UK

${ }^{5}$ Department of Physiotherapy, Sheffield Teaching Hospitals NHS Trust, Royal Hallamshire Hospital, Sheffield, UK

${ }^{6}$ Department of Orthopaedics \& Trauma, Sheffield Children's Hospital NHS Foundation Trust, Birmingham, UK

${ }^{7}$ Maastricht University Medical Centre, Maastrict, The Netherlands

${ }^{8}$ Department of Orthopaedic Surgery Inselspital, University of Berne, Berne, Switzerland

Acknowledgements Our thanks to Julie Sterling and Marie Morley for providing their experience as patients following LSFS to the design and detail of this study.

Contributors AR is the chief investigator leading protocol development, approvals, analyses and dissemination. AR, JBS, MV, AC, PW, LB and NRH have led on conception, design and are overseeing data analysis, interpretation and synthesis of findings into parallel studies. NRH is the lead for patient involvement. AS will oversee the qualitative methods and data analysis. $A E$ and MR are the principal investigators at the two clinical sites. AM has finalised the topic guide and diary and will be carrying out data collection. AM, AR and AS will be involved in data analysis. All authors have contributed to methodological decisions. All authors will contribute to data interpretation, conclusions and dissemination. AR drafted the initial manuscript. All reviewers have read, contributed to and agreed the final manuscript. $A R$ is the guarantor.

Funding This work was supported by Chartered Society of Physiotherapy Charitable Trust Physiotherapy Research Foundation, grant number PRF-16-A21.

Competing interests None declared.

Ethics approval NHS ethical approval, HRA approval and individual site confirmation of capacity and capability have been obtained (IRAS 223283).

Provenance and peer review Not commissioned; peer reviewed for ethical and funding approval prior to submission.

Open Access This is an Open Access article distributed in accordance with the Creative Commons Attribution Non Commercial (CC BY-NC 4.0) license, which permits others to distribute, remix, adapt, build upon this work non-commercially, and license their derivative works on different terms, provided the original work is properly cited and the use is non-commercial. See: http://creativecommons.org/ licenses/by-nc/4.0/

(c) Article author(s) (or their employer(s) unless otherwise stated in the text of the article) 2018. All rights reserved. No commercial use is permitted unless otherwise expressly granted.

\section{REFERENCES}

1. Gibson JN, Waddell G. Surgery for degenerative lumbar spondylosis: updated Cochrane Review. Spine 2005;30:2312-20.

2. online HES. HaSCl C, ed. Procedure and Intervention: 3 character tables. 16, 2016. http://www.hesonline.nhs.uk. (Accessed 28 jul 2016).

3. Koenders N, Rushton A, Heneghan N, et al. Pain and disability following first-time lumbar fusion surgery for degenerative disorders: a systematic review protocol. Syst Rev 2016;5:72.

4. Weinstein JN, Lurie JD, Olson PR, et al. United States' trends and regional variations in lumbar spine surgery: 1992-2003. Spine 2006;31:2707-14.

5. NICE. Low back pain and sciatica in over 16s: assessment and management, NICE guideline [NG59: National Institute for Health and Care Excellence,, 2016.
6. Willems $\mathrm{P}$, de Bie R, Oner C, et al. Clinical decision making in spinal fusion for chronic low back pain. Results of a nationwide survey among spine surgeons. BMJ Open 2011;1:e000391.

7. Rushton A. Survey of current surgeon practice for patients undergoing lumbar spinal fusion in the UK. World Journal of Orthopaedics 2015;6:483-90.

8. Stromqvist B, Fritzell P, Hagg O, et al. Follow-up of Lumbar surgery in sweden 2007, the swedish national spine register. The Swedish Spinal Surgery Society 2007.

9. Mannion AF, Brox JI, Fairbank JC. Comparison of spinal fusion and nonoperative treatment in patients with chronic low back pain: long-term follow-up of three randomized controlled trials. Spine $J$ 2013;13:1438-48.

10. Rushton A, Eveleigh G, Petherick EJ, et al. Physiotherapy rehabilitation following lumbar spinal fusion: a systematic review and meta-analysis of randomised controlled trials. BMJ Open 2012;2:e000829.

11. Greenwood J, McGregor A, Jones F, et al. Rehabilitation following lumbar fusion surgery: a systematic review and meta-analysis. Spine 2016;41:28-36.

12. Rushton A, Wright C, Heap A, et al. Survey of current physiotherapy practice for patients undergoing lumbar spinal fusion in the United Kingdom. Spine 2014;39:1380-1387.

13. Smith JE OM. Interpretative phenomenological analysis. Smith JA, eds. Qualitative psychology: a practical guide to methods London Sage, 2008.

14. Smith JA FP, Larkin M. Interpretative phenomenological analysis: theory, method and research. London: Sage, 2009.

15. Rodham K, Fox F, Doran N. Exploring analytical trustworthiness and the process of reaching consensus in interpretative phenomenological analysis: lost in transcription. Int J Soc Res Methodol 2015;18:59-71.

16. Tong A, Sainsbury P, Craig J. Consolidated criteria for reporting qualitative research (COREQ): a 32-item checklist for interviews and focus groups. Int J Qual Health Care 2007;19:349-57.

17. Do H. Equity and excellence: liberating the NHS. Do H, ed. London,UK: The Stationery Office Limited, 2010.

18. Beaton DE, Clark JP. Qualitative research: a review of methods with use of examples from the total knee replacement literature. $J$ Bone Joint Surg Am 2009;91:107-12.

19. Ja S. Evaluating the contribution of interpretative phenomenological analysis. Health psychology review 2010;5:9-27.

20. Dale O, Hagen KB. Despite technical problems personal digital assistants outperform pen and paper when collecting patient diary data. J Clin Epidemiol 2007;60:8-17.

21. Rushton A, Heneghan NR, Heap A, et al. Patient and physiotherapist perceptions of rehabilitation following primary lumbar discectomy: a qualitative focus group study embedded within an external pilot and feasibility trial. BMJ Open 2017;7:e015878.

22. Gibson BE, Mistry B, Smith B, et al. The Integrated use of audio diaries, photography, and interviews in research with disabled young men. Int J Qual Methods 2013;12:382-402.

23. Brocki JM, Wearden AJ. A critical evaluation of the use of interpretative phenomenological analysis (IPA) in health psychology. Psychol Health 2006;21:87-108.

24. Biggerstaff D, Thompson AR. Interpretative phenomenological analysis (IPA): a qualitative methodology of choice in healthcare research. Qual Res Psychol 2008;5:214-24.

25. Ja S. Reflecting on the development of interpretative phenomenological analysis and its contribution to qualitative research in psychology. Qualitative Research in Psychology 2004;1:39-54.

26. Staniszewska S, Brett J, Simera I, et al. GRIPP2 reporting checklists: tools to improve reporting of patient and public involvement in research. Res Involv Engagem 2017;3:13. 